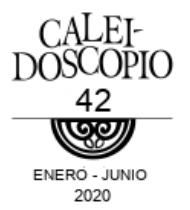

\title{
El malestar en los hombres: una revisión de alcances
}

Discomfort in men: a scoping review

CAROLINA OLVERA CASTILLO

MARÍA GABRIELA LUNA LARA

Universidad de Guanajuato, México

\section{RESUMEN}

El malestar es un tema en pleno desarrollo desde los estudios de las masculinidades y actualmente es un terreno fértil para seguir generando líneas de investigación. El presente trabajo siguió la metodología de scoping review cuyo objetivo fue explorar el alcance de los artículos y textos académicos sobre las masculinidades en relación con el malestar. Los resultados apuntan hacia una conceptualización más sólida del malestar como un estado entre la salud y la enfermedad, cuyas causas han sido identificadas como conflictos y pérdidas con relación al (in)cumplimiento de los mandatos de la masculinidad hegemónica. Sus consecuencias repercuten directamente en la salud física y mental. Las conclusiones se centran en la reflexión de la posibilidad del malestar como una punta de flecha para el cambio en las relaciones de género.

Palabras clave: malestar; masculinidades; masculinidad hegemónica; Latinoamérica. 


\section{ABSTRACT}

The issue of discomfort is a subject in full development from the studies of masculinities and is currently a fertile ground to continue generating lines of enquiry, although it is not exempt from criticism and inaccuracies. The present work followed the methodology of scoping review whose aim was to explore the scope of articles and academic texts regarding masculinities in relation to discomfort. The results point to a conceptualization of discomfort as a state between health and illness, whose causes have been identified as conflicts and losses in relation to (non)fulfilment of the mandates of hegemonic masculinity. Its consequences have a direct impact on physical and mental health. The conclusions focus on the reflection of the possibility of discomfort as a watershed for the change in gender relations.

Keywords: discomfort; masculinities; hegemonic masculinity; Latin America.

Pareciera inevitable que, al hablar de masculinidad hegemónica $(\mathrm{MH})$, también debemos hablar del malestar. La MH es entendida como un modelo identitario que dota de privilegios y también supone costos; por un lado, es una estima de todo aquello que, valida la ejecución del poder, la productividad, la virilidad y la fortaleza siempre en contraste con la otredad, todo aquello que no es hombre, sea lo que eso fuere: mujeres u otros hombres que se sienten incómodos con la hegemonía, ya sea porque no pueden o porque no quieren alinearse a ella. La constante necesidad de validación de esta masculinidad exige su reafirmación a lo largo de la vida de los hombres; quienes en este proceso se desgastan, paradójicamente disminuyendo su 
salud en esa búsqueda de bienestar que supondría el cumplimiento de los requisitos del modelo hegemónico; siendo potencialmente mortal para ellos mismos y quienes los rodean.

¿Qué ocurre cuando la masculinidad hegemónica se topa con la diversidad en las familias y de género, la precariedad y las exigencias de justicia social? Las evidencias apuntan hacia una crisis de la masculinidad o en la masculinidad (Bonino, 2008; Figueroa, 2007; Tena, 2014), que ha sido uno de los puntos de partida para identificar un concepto ya documentado con más amplitud en el caso de las mujeres: el malestar (Burin, 2010; Figueroa, 2007; Tena, 2007; Tena y Jiménez, 2014).

Con la intención de adentrarnos en el tema, nos hemos hecho la siguiente pregunta: a través de la literatura existente ¿qué se conoce sobre el concepto de malestar en los hombres desde el estudio de las masculinidades? Y para responderla nos fijamos el objetivo de examinar el alcance de los artículos y textos académicos sobre las masculinidades en relación con el malestar.

Para ello, se realizó una revisión sistemática exploratoria o por su nombre en inglés: scoping review (Arksey y O'Malley, 2007). Este procedimiento metodológico consistió en que, una vez determinada la pregunta de investigación se procedió a identificar los estudios que potencialmente nos pudieran servir. La búsqueda de documentos comenzó en enero de 2018 y terminó en febrero de 2019 y la ventana de búsqueda abarcó elementos que datan de 1993 hasta 2019.

Se utilizaron las siguientes bases de datos y repertorios de acceso libre: Citas Latinoamericanas en Ciencias Sociales y Humanidades, Dialnet, Directory of Open Acces Journals, EBSCO, Elsevier ScienceDirect, Elsevier Scopus, GALE, Red Iberoamericana de Innovación y Conocimiento Científico, Redalyc y SciElo. También se utilizaron recursos como Google Académico, listas de referencias y páginas de intercambio académico como Academia y Research Gate. 
La búsqueda se centró en textos exclusivamente en español puesto que favorece la comprensión de los mismos para ambas autoras cuyo idioma natal es éste mismo. A su vez el español se habla y se publica en numerosas regiones de Latinoamérica. Además la búsqueda se restringe a otros idiomas debido a los recursos limitados de esta investigación y los costos que supondría hacer traducciones certificadas de otras lenguas que se hablan en América Latina como el portugués o el francés. Por otro lado, se buscaron textos centrados en esta región para poder comprender la zona geográfica que nos concierne por semejanza sociohistórica-política y cultural.

Los conceptos clave de búsqueda fueron: masculinidad y malestar. Las primeras exploraciones se hicieron con las palabras: masculinidad, malestar, hombres, paternidades y género, siendo alternados en cada entrada, sin embargo, se obtuvieron resultados limitados. Debido al interés de las autoras por el tema de las paternidades, se incluyó esta palabra en las indagaciones preliminares, puesto que esta revisión inicialmente se contempló como un sondeo del concepto del malestar en la paternidad. No obstante, notamos la escasez de trabajos centrados en hombres que son padres y que además tratan el malestar, por ello se decide reducir el número de palabras clave con lo que se ampliaron los resultados.

Después se hizo la selección de los textos que cumplian con las características anteriormente descritas, además de haber sido leídos en su totalidad para determinar su relevancia de inclusión, se encontraron 41 elementos: 24 artículos, 11 capítulos de libro, dos tesis, dos libros y un documento de trabajo.

El siguiente paso fue sintetizar el material clasificándolo según los siguientes temas: conceptualización del malestar, su relación con la masculinidad hegemónica, las causas que hasta ahora se han identificado como fuentes de malestar, algunos efectos y las reflexiones hacia las oportunidades de cambio de las relaciones de género. El paso final del procedimiento fue que, 
conforme a este ordenamiento, se compilaron y resumieron los estudios para ser reportados como a continuación se presenta.

\section{EL MALESTAR COMO CONCEPTO}

El malestar está insistentemente presente en las relaciones de género, es justamente del malestar generado por las situaciones de desigualdad que los movimientos feministas han denunciado y exigido la eliminación de la discriminación que viene de la mano de las desigualdades. Esta experiencia en mujeres no solo ha sido expresada, sino construida y comprendida, constituyendo una de las raíces del propio feminismo (Tena, 2010). El malestar en los hombres no es un tema reciente, sin embargo, sí lo es su conceptualización y problematización desde el enfoque de los estudios de género, especialmente desde el enfoque de las masculinidades.

El que los hombres reflexionen y se hagan el propósito de entender la agenda feminista desde sus propias identidades, cuestionando su posición de poder, refleja el inicio de otro cambio en las relaciones de género; a este movimiento podemos llamarle estudios de las masculinidades. Por sí solos tienen distintas vertientes, que van desde los temas de salud y paternidades hasta los de diversidad sexual. Sin embargo, el tópico que ahora nos interesa desentrañar es el del malestar masculino.

El malestar masculino no está exento de controversias, antes de abordar el tema como tal, debemos recordar que, al hacer un reconocimiento del malestar en los hombres, a las autoras y autores no se les está olvidando la posición de privilegio que todavía ostentan los hombres (Figueroa, 2014; Jiménez, 2016; Tena, 2007). Se trata de examinar y nombrar dichos malestares, sin victimizar a los hombres, que de entrada posiblemente no se hayan dado cuenta del malestar como tal (Figueroa, 2013, 2014). 
En los primeros textos que hacen alusión al malestar, éste ha sido asociado con distintas palabras: molestias, quejas, tensiones, dificultades, contradicciones, incomodidad, desazón o frustraciones. Todas ellas aluden a un algo que no está claro y que difícilmente es nombrado por quienes lo padecen. Por lo que en los estudios revisados encontramos que el malestar es definido por su propia imprecisión. Es una expresión ambigua, en donde no se está por completo mal, que de hecho puede estar en presencia de salud ya que no alude directamente a una enfermedad, pero es un estado en el que el bienestar está disminuido (Jiménez y Tena, 2007; Tena, 2007, 2014) y por si fuera poco, es casi imperceptible. Incluso si los hombres tienen clara la situación que provoca la incomodidad, rara vez identifican el malestar como tal (Bonino, 2000; Figueroa, 2001; Olavarría, 2001; Pallahuala, Avendaño, y Estrada, 201l) ya que pueden seguir viviendo y funcionando (Burin, 2010) casi con normalidad.

Resalta la cualidad transicional del concepto, como un estado en medio de lo subjetivo y lo objetivo, lo interno y lo externo o el punto medio entre la salud y la enfermedad mental interactuando en una misma persona (Burin, 2007, 2010; Cantoral, 2014). El malestar es un sufrimiento o padecimiento más subjetivo que irrumpe en la dicotomía de salud-enfermedad ya que no toma parte en ninguno de estos términos ni tampoco puede ser definido por ellos (Burin, 2010). La salud entendida más allá de la ausencia de enfermedad, incluyendo tanto lo físico como mental en la búsqueda, mantenimiento y recuperación del equilibrio fisiológico y/o emocional, que además es socialmente construido e imaginado (Figueroa, 2014); de tal manera que el concepto de salud construido por los hombres es diferente al de las mujeres como consecuencia de sus aprendizajes de género. El autocuidado y el mantenimiento de la salud no juegan un papel importante en la construcción de la identidad masculina (De Keijzer, 2006); los patrones de vida aprendidos en los hombres reflejan una socialización autodestructiva de sus 
cuerpos y abandono de sus emociones, incluso rechazando su atención o búsqueda de ayuda ante el malestar o la enfermedad (Juárez y Herrera, 2016) porque eso sería aceptar la fragilidad (Figueroa, 2007).

En primera instancia el poder reconocer el malestar es una tarea un tanto más difícil para algunos hombres ya que no se identifican ausencias emocionales, ansiedad o tristeza (Figueroa, 2001). Si llegasen a reconocer el malestar puede que lo nieguen (Jiménez, 2016) o lo verbalizan poco o nada (Fleiz, Ito, MedinaMora, y Ramos, 2008; Tena y liménez, 2014) en comparación con las mujeres, puesto que los sitúa en una posición difícil que está asociada al género (Salguero, Córdoba, y Sapién, 2014; Salinas, 2007; Salinas y Arancibia, 2006).

El que les resulte complicado ponerlo en palabras es resultado del conflicto emocional asociado a las relaciones de poder, ya que el sistema patriarcal les pide no sentir aquellas emociones que son generadas por el mismo sistema (Connell y Messerschmidt, 2005); estos aprendizajes que los presupone como poco emocionales, fuertes, sin debilidades y con pocas necesidades afectivas (Rocha, 2016). A lo largo de sus historias, los hombres han crecido con el refuerzo a actitudes que los pueden poner en riesgo físicamente a la vez que crecen en el descuido de sus emociones, exigiendo la represión de sus miedos y en caso de llegar a sentirlos, se les pide silenciarlos; por lo que, el reconocimiento de los malestares supondría una muestra de debilidad, que se contradice con la masculinidad tradicional.

De esta contradicción surge otro planteamiento del malestar, uno que lo coloca como el resultado de la contraposición del querer y el deber; propone el surgimiento del malestar desde un punto de vista freudiano, como el constante conflicto entre la voluntad de la masa y la libertad individual (Cantoral, 2014). En otras palabras, es la propuesta de observar el malestar como el punto de quiebre entre los mandatos de la masculinidad hegemónica y lo que realmente puede ser cumplido de estos 
mandatos en vista de los cambios económicos, la diversificación de las configuraciones familiares y evidentemente de los cambios en las relaciones de género.

\section{LA MASCULINIDAD HEGEMÓNICA, UNA PRECONDICIÓN PARA EL MALESTAR}

En contraposición a la feminidad, la masculinidad existe y se comprende como una construcción histórica, cultural e incluso geográfica que configura diversas prácticas que interactúan en un sistema de relaciones de género (Connell, 1997). Estas relaciones están usualmente en tensión ya que dentro del sistema entran en juego la interacción de la subordinación y la dominación; siendo el orden patriarcal el que ejerce la dominación. No solo se habla de subordinación de mujeres hacia hombres, sino de un orden específico de género que es el de la masculinidad hegemónica, que es la base de construcción de las identidades masculinas (Bonino, 2002); obedeciendo a un orden jerárquico de raza, clase y/o disidencia-diversidad sexual (Connell, 1997; Connell y Messerschmidt, 2005).

Ésta puede ser embestida solo por unos cuantos hombres, pero de la cual se sirven todos ellos. Incluso, como lo señala Segato (2018), "el orden patriarcal..a veces está encarnado por mujeres" (s.p.), internalizando en nosotras mismas sentimientos desdeñosos hacia lo femenino, en ocasiones, adoptando formas masculinizadas que en apariencia nos benefician. Por ejemplo, algunas las mujeres a cargo de grupos o empresas que prefieren que se les diga jefe en vez de jefa, o que su propia vestimenta y forma de actuar reflejan lo masculino, lo de mayor estatus dentro del sistema patriarcal. Aunque también es una forma de sobrellevar los posibles ataques de este orden de género hacia sus cuerpos.

Al suponer que existe una masculinidad hegemónica, existirán vivencias y prácticas que quedarán excluidas de ella, 
éstas son lo que algunos llaman masculinidades alternativas, que al no ser hegemónicas están subordinadas a la primera (Bonino, 2002; Connell y Messerschmidt, 2005; Núñez, 2004). La condición de dominación por lo general requiere del ejercicio de autoridad, e incluso de violencia, llegando a ser usada por sus integrantes privilegiados para reafirmar su masculinidad de tal manera que se sienten plenamente justificados por el sistema patriarcal (Connell, 1997); aunque la dominación no siempre es forzosa, también puede ser a través de acuerdos culturales o por marginación de las masculinidades alternativas (Connell y Messerschmidt, 2005).

La hegemonía como dominación cultural, cambia conforme al momento histórico y lugar en el que se despliega (Bonino, 2002), siempre tendiente a estabilizar o reconstruir las condiciones para el ejercicio del poder (Connell y Messerschmidt, 2005). Lo que hoy en día conocemos como masculinidad hegemónica, puede que no haya sido tal sino hasta el Renacimiento, aunque sigue manteniendo sus elementos más básicos (Connell, 1997) que le permiten estabilizarse como referente de la identidad masculina (Bonino, 2002). Ni las masculinidades ni las feminidades son estáticas, su transformación es producto quiebres históricos y contradicciones internas (Connell, 1997), aunque el proceso no es necesariamente rápido (Madrid, 2017). Los cambios estructurales en la sociedad suelen ser lentos, graduales y en cuestiones de género: desiguales, porque son transiciones que están mediadas por las creencias y valores que aún son vigentes (Piña, 2003)

No es que la masculinidad hegemónica deje de existir en un futuro, más bien será otra que transmute para adaptarse a las realidades culturales, manteniendo algunos de sus elementos, pero incorporando otros nuevos que le permitirán subsistir (Bonino, 2002; Connell y Messerschmidt, 2005). Los elementos incorporados de las masculinidades no hegemónicas son una estrategia que, en lugar de invalidarlas, las convierte en lo 
deseable, aunque sigue existiendo la opresión hacia estas formas de masculinidad puesto que "en la práctica, tanto la incorporación como la opresión pueden ocurrir juntas" (Connell y Messerschmidt, 2005, p. 848,traducción propia), de tal manera que las experiencias de un mismo grupo de personas pueden oscilar desde la más extrema violencia hasta la validación de su estilo de vida.

En el caso del cambio de las expectativas hacia la masculinidad, lo que pudo haber sido lo admirable ya no es la masculinidad invulnerable, sino una más sensible, aunque todavía se espere que el hombre sea lo más racional posible, lo cual puede llegar a ser una contradicción en el sistema de género inflexible. Las contradicciones y conflictos en este sistema repercuten de manera especial en los hombres, cuyo referente de identidad es el de la masculinidad hegemónica. Las exigencias de ser un proveedor económico, de recursos, material y de seguridad sigue siendo plausible y se mezcla con aquellas expectativas que van más allá de lo que la sociedad le habilita a ser y hacer. Incluso si se espera que los padres, especialmente los más jóvenes (Herrera, Aguayo y Goldsmith, 2018), sean más involucrados, cariñosos y activos, el mismo patriarcado los inhabilitó para sentir emociones que el mismo sistema creó (Connell y Messerschmidt, 2005); debido a sus aprendizajes puede que algunos no estén capacitados para responder a las demandas afectivas, incluso si lo intentan; queriendo cumplir con el mandato de buen padre, sea lo que eso signifique en la actualidad, pero sin ver los resultados esperados, generando así malestar por el ejercicio de su masculinidad.

En la actualidad, en un mundo en donde es posible enteramos en unos cuantos minutos de lo que sucede en distintas partes del mundo, es posible observar una escalada en la frecuencia y formas de violencia a nivel global, lo que puede ser un indicador de crisis en el orden de género contemporáneo (Connell y Messerschmidt, 2005). Aunque Connell $(1997,1998)$ 
señala que la masculinidad como tal no puede entrar en crisis puesto que no es un sistema, si se puede hablar de la crisis del orden sexista de género actual. Para otras autoras y autores que si utilizan el término de crisis de la masculinidad hegemónica (Badinter, 1993; Burin, 2007; Jiménez y Tena, 2007; Montesinos, 2004; Solís, 1998; Tena, 2007), les queda claro que está en un punto de quiebre para la transformación, no sin antes generar condiciones propicias para el malestar masculino. Esto no significa que los hombres sean víctimas de las condiciones más igualitarias que han ido ganando las mujeres, aunque tampoco los define como victimarios (Jiménez y Tena, 2007), su malestar más bien, es una consecuencia del choque entre el sistema patriarcal que valida la masculinidad hegemónica y la resistencia hacia el cambio que desautorice el ejercicio de su poder.

\section{PÉRDIDAS Y CONFLICTOS: CAUSAS DEL MALESTAR}

Las soluciones que antes ofrecía la masculinidad hegemónica ahora no sirven del todo para responder a las demandas sociales actuales, como las del sistema económico globalizado (Batthyány, 2013; Tena, 2007) y las reconfiguraciones familiares, enfrentándose a un posible colapso de la legitimidad del poder patriarcal (Connell, 1997). En parte esta perturbación se debe al constante desafío de los movimientos emancipatorios de las mujeres (Connell y Messerschmidt, 2005; Jiménez, 2008) y los colectivos que buscan la democratización de las relaciones de género, quienes tensionan la continuidad de la rigidez en los roles sexuales, especialmente en los que tienen que ver con lo laboral y familiar. Si a esto se le suma la precarización del empleo o la pérdida de éste (Jiménez, 2008), más las pocas estrategias de adaptación tanto personales como institucionales (Jiménez, 2008; Mena, 2015), se tiene las condicionantes propicias para el malestar. 
Solís (1998) teoriza que la feminización de la ciencia, la tecnología, la política, el ejército y sobre todo, el empleo remunerado, ha generado efectos no deseados en los hombres, de tal manera que se vulneran dos ejes centrales de la identidad masculina hegemónica: la autoridad y el papel de proveedor (Montesinos, 2004; Rojas, 2017). Actualmente los ingresos de las mujeres no son extra, en un contexto de crisis económica global, sus ingresos son claves para el mantenimiento de la economía (Gálvez, 2013). Su incursión al mercado laboral ha mejorado las expectativas económicas de las familias (González y Jurado, 2015), aunque también ha generado inestabilidad en los beneficios (Connell, 1997) que a los hombres les genera figurar en el campo del trabajo remunerado: no solo lo económico, también en lo identitario.

Aunque no solo por la presión externa de mayor democratización de las relaciones de género, también sienten la presión de sus parejas para modificar las relaciones en la familia para que colaboren en igual o mayor medida en las labores domésticas y de crianza de sus hijas e hijos (Abarca, 2000; Ramos, 2001). Incluso, cuando un mismo hombre esté a favor la justicia social, puede tener sentimientos de ambivalencia al observar los proyectos de cambio en los que oscila entre la aceptación y el rechazo de la igualdad de género (Connell y Messerschmidt, 2005).

La palabra clave en los trabajos sobre crisis de la masculinidad es la de conflicto. La discontinuidad de los roles de género tradicionales y las nuevas expectativas de conductas igualitarias son fuente de malestar en los hombres, lo aprendido pierde vigencia (Téllez y Verdú, 2011) y lo nuevo está en plena formación, creando incertidumbre con respecto a sus comportamientos. El contexto actual impide la justificación de comportamientos inscritos en la autoridad que va perdiendo reconocimiento (Montesinos, 2004). Por ejemplo, el dilema entre una paternidad más afectiva o una más autoritaria; en el estudio 
de Villanueva (2015), con padres que acuden a terapia, se observa cómo se enfrenta a dificultades para poder ejercer su autoridad que se ve censurada, lo cual les causa un conflicto, aunado a las demandas afectivas que los hijos e hijas formulan. Demandas que no siempre pueden cumplir puesto que los padres no fueron educados para ello, reprimiendo y controlando sus sentimientos (Figueroa, 2014; Olavarría, 2001; Pallahuala et al., 2011) y generando así un malestar en quienes se alejan (quieran o no) del modelo hegemónico, pero que no tienen un referente sólido sobre otra forma de ser hombres y padres.

Otro concepto con el que nos topamos en nuestra revisión fue el de la pérdida (Burin, 2007; Fleiz et al., 2008; Jiménez y Tena, 2007; Montesinos, 2004; Perujo, 2015; Tena, 2007; Tena y Jiménez, 2014; Tovar, 2016), el malestar no solo se deriva del conflicto de las desigualdades de género (Burin, 2010), también surge de los vacíos que no pueden ser llenados por ella, dejándolos a la suerte de aguantar y no mostrar debilidad si se llegan a enfrentar al abandono o la soledad (Fleiz et al., 2008). Los hombres experimentan pérdidas en algunos elementos de la identidad masculina (Montesinos, 2004), que básicamente son el estatus, el control, la autoridad, los privilegios o el poder (Jiménez, 2008; Tena, 2007; Tena y Jiménez, 2014; Tovar, 2016), cuyo origen es la pérdida de la salud, del trabajo o la familia (Fleiz et al., 2008; jiménez y Tena, 2007).

Es imprescindible comprender que algunas pérdidas están estrechamente relacionadas con deterioro de la economía: la pérdida y precarización del trabajo que a su vez puede resultar en pérdidas de la salud y la pérdida de relaciones familiares al verse obligados a buscar empleo en el extranjero. Tena y Jiménez (2007) explican concretamente la disminución y pérdida del trabajo debido a factores como:

.la instauración de un modelo de desarrollo neoliberal caracterizado por una economía abierta, competitiva y regulada 
por el mercado con una cada vez menor participación del Estado en sus funciones económicas; transformaciones tecnológicas y organizacionales que buscan la disminución de costos no obstante la disminución de la planta laboral y las condiciones de empleo; quiebras de empresas incapaces de ajustarse a las nuevas condiciones de competencia desigual e incremento de las importaciones por la globalización de la economía; privatización de industrias estatales y servicios públicos y adelgazamiento de servidores públicos sustituidos por empresarios con nula experiencia en el sector... (p. 15)

La pérdida del empleo o la simple idea de perderlo ya representa una amenaza para el cumplimiento de los mandatos hegemónicos éxito y proveeduría (Tena, 2007), especialmente en los hombres que son padres y que tienen una responsabilidad diferente a la de los hombres solteros. Es cierto que la paternidad por sí sola genera algunos malestares en su ejercicio, sin embargo ante una separación importante, el malestar aumenta (Burin, 2007), llegando incluso a salir del rango de un malestar para convertirse en enfermedad como tal. De ello nos hablan Torres y Rodríguez (2019), en donde el malestar específico en hombres que emigraron es el derivado de la obligación de proveer y que no sea suficiente, a la vez que intentan establecer y mantener vínculos afectivos a distancia. El incumplimiento de estos mandatos puede desembocar en el mejor de los casos en un malestar, sin embargo, es usual que se desaten problemáticas psicológicas más graves.

Otro ejemplo es el de las familias en donde el padre se hace cargo casi exclusivamente de sus hijos e hijas sin la presencia de la madre, resultando en malestares derivados de la pérdida de la pareja y después de la pérdida de control del arreglo de género preestablecido, teniendo dificultades para conciliar lo laboral y lo familiar, o para aprender a realizar labores del hogar y cuidados de los hijos e hijas. En algunos casos no lograron superar esas barreras de género, dejando a sus redes de apoyo a cargo de algunas de estas tareas, tomando en cuenta que sus 
redes usualmente están conformadas por mujeres (Aguayo, Barker y Kimelman, 2016; Mena y Rojas, 2010). Otro es el caso de los padres divorciados, en donde además de vivir la separación de sus hijos e hijas, sienten la pérdida de tiempo para con ellos al no estar presentes (Perujo, 2015).

\section{LOS RECUENTOS DEL MALESTAR}

Aunque el malestar no es sinónimo de enfermedad y mucho menos es igual a una muerte inminente, si es un antecedente de ambas (Figueroa, 2014; Tena y Jiménez, 2007), ya que propio ejercicio de la masculinidad hegemónica es un factor de riesgo para los mismos hombres, sea ellos mismos u otros, para las mujeres, las niñas y niños (Bonino, 2000; De Keijzer, 2006; Tena, 2007; Tuñón y Bobadilla, 2005). La siguiente frase resulta oportuna para comenzar a escribir sobre los hallazgos de las investigadoras e investigadores: "El ideal de autosuficiencia en la que se basa la masculinidad tradicional, suele armonizar con el auto concepto de forma positiva, pero puede oponerse a la salud mental o a la integridad física" (Pallahuala et al., 2011, p. 74).

La disminución de salud física y mental es la condición más evidente en los hombres que sufren de malestares, sin embargo no siempre suelen ser conscientes de los factores que los propician y por lo tanto no pueden resolverlo de manera inmediata (Jiménez, 2013; Tena, 2007), es solo hasta que sienten afectada su vida cotidiana que el malestar adquiere sentido (Juárez y Herrera, 2016).

Anteriormente se ha mencionado que la socialización de la masculinidad tradicional les impide a muchos hombres buscar el cuidado del propio cuerpo, minimizando su mantenimiento e incluso rechazando la ayuda que necesitan. El cuerpo puede verse implicado con dolores musculares, problemas gástricos, desgaste y agotamiento físico, sensación de desgano y alteraciones cardiovasculares (Fleiz et al., 2008; Juárez y Herrera, 
2016; Sapién, Salguero y Córdoba, 2011; Tena, 2007, 2014), al menos hasta donde se ha registrado.

Cada hombre vive diferente la pérdida de guía o sentido y aunque todos sienten dolor (Abarca, 2000), se demuestra de distintos modos a través de alteraciones psicológicas que hablan por ellos a través de problemas de sueño, manifestación de sentimientos de ira, arrepentimiento, angustia, desinterés laboral, soledad (voluntaria e involuntaria), tristeza, enojo, aislamiento, alienación, disminución del deseo sexual, sensación de derrota, depresión, inseguridad (Bonino, 2002; Figueroa, 2001; Fleiz et al, 2008; Olarte, 2016; Ramos, 2001; Rocha, 2016; Tena, 2007, 2014). Usualmente estas dificultades se canalizan a través de la evasión (Morales, Catalán y Pérez, 2018), aunque otras veces explotan, observándose un incremento de las conductas violentas.

Es de esperarse que las tensiones que desatan el malestar no solo tengan consecuencias en la propia salud de los varones, también repercuten en quienes los rodean. Sea por pérdida de autoridad o conflictos internos, las autoras y autores han notado que el malestar se recicla a través de la ira y la frustración (Jiménez, 2013, 2016) y está asociado a comportamientos violentos que algunos hombres ejercen contra sus cónyuges (De Keijzer, 2006; Montesinos, 2004; Tena y Jiménez, 2014), en un intento de "reestablecer el orden de género" (Rojas, 2017, p. 82). Las conductas autodestructivas y el uso de la violencia son una expresión mal encaminada de las frustraciones, tristeza o la soledad que los hombres sienten pero que reprimen (Figueroa, 2014; Tena y liménez, 2007).

Desde la otra cara de la moneda, las investigaciones con un enfoque mayormente cuantitativo son realizadas en la perspectiva del bienestar o de búsqueda de las condiciones que mejoren los malestares de los hombres. En cierto modo, estos corroboran lo que la teoría y las entrevistas señalan: existe un rechazo a la percepción de rasgos negativos de la masculinidad 
hegemónica, al menos a nivel de discurso políticamente correcto.

En la investigación de Rocha (2016) se observó que si los hombres se definían a sí mismos con rasgos negativos de la masculinidad (violencia, agresividad, orgullo, etc.) había mayor presencia de depresión y soledad, sin embargo también se arrojaron resultados similares si se describían con rasgos asociados a la feminidad estereotípica (vulnerabilidad, sumisión, pasividad, etc). Lo que indica que, si bien no se quieren identificar con una parte de la masculinidad hegemónica, siguen percibiendo lo femenino como algo indeseable. Pallahuala, Avendaño y Estrada (2011) obtuvieron resultados similares y además, encontraron que si los hombres se definen a sí mismos con rasgos masculinos y femeninos más equilibrados existe mayor bienestar.

También se ha encontrado que si los hombres expresan más sus sentimientos y tienden a ser más empáticos, las sensaciones de ansiedad y malestar son menores que en quienes no lo hacen (Nieri, 2017). El expresar más la afectividad les favorece al hacer frente a situaciones problemáticas (Olarte, 2017). Probablemente estos varones lleven a cabo un proceso más acertado de reconocimiento de los malestares a modo de reflexión de su cotidianeidad dependiendo de sus condiciones de vida (Abarca, 2000; Cantoral, 2014). En el apartado siguiente revisaremos las ideas que en general abogan por la expresión del malestar como catalizador de transformaciones en el orden de género.

\section{UNA VENTANA AL CAMBIO}

En este último apartado a manera de cierre, se relatarán las ideas de las y los investigadores que han reflexionado sobre la posibilidad de cambio, la masculinidad hegemónica y el malestar como elementos relacionados. La primera idea se centra en la 
reconceptualización de la masculinidad hegemónica como la clave del cambio del sistema de género. Connell y Messerschmidt (2005) lo predicen como una hegemonía positiva, que avale las relaciones de género democráticas, inhabilitando las diferencias en el ejercicio del poder. Para ello la resignificación simbólica de la masculinidad es imprescindible para hacer tangible de las transformaciones en las prácticas sociales, de lo contrario se queda solamente en el discurso (Montesinos, 2004). Por otro lado, para Burin (2010) en vez de una oportunidad de transformación, existe la posibilidad de que el cambio más bien genere una posible catástrofe subjetiva, dependiendo de la adhesión de los varones al modelo de masculinidad tradicional.

La segunda idea es el cuestionamiento de los aprendizajes de conductas autolesivas y dañinas hacia su entorno (Figueroa, 2007). Si se comenzara por identificar aquello que está ausente y tratar de sentir melancolía por ello, se puede habilitar otra forma de vivir como hombres, resignificándose como afectivos, más corresponsables (Figueroa, 2001), equitativos y democráticos (Salguero et al., 2014). Badinter (1993) habla de un hombre reconciliado, ¿reconciliado con qué?, con las partes femeninas que en la actualidad son rechazadas e incluso temidas, aquel que amalgama la solidez y la sensibilidad. Un camino estratégico es el de la paternidad que como un proceso de constante aprendizaje (Salguero et al., 2014) resulta más flexible y presenta menor resistencia al cambio (Ramírez y Uribe, 2008), se puede replantear su ejercicio hacia uno que no tenga que dañar la figura materna para sostenerse a sí misma (Badinter, 1993).

Como tercera idea: el malestar interviene como incomodidad y molestia constante, generando la reconfiguración de las prácticas de género (Cantoral, 2014). Un paso posterior a la identificación es su verbalización o expresión por parte de los hombres que lo padecen (De Keijzer, 2006; Olarte, 2017; Tena, 2014), después habrá quienes realicen el proceso de reflexión 
sobre aquello que les aqueja (Cantoral, 2014) y del por qué afecta su vida cotidiana; habrá también algunos que se replanteen de manera crítica el ejercicio de su masculinidad, siendo a su vez cómplices de otros hombres "en la búsqueda de nuevos ejercicios de la masculinidad" (Figueroa, 2007, p. 379).

Tras la lectura de los trabajos puede concluirse en primer lugar que todavía son un área de oportunidad para los estudios de las masculinidades, priorizando los matices de las relaciones entre el malestar-empleo y el malestar-salud, que son los temas privilegiados por las y los investigadores. Después, la mayoría de los estudios fueron realizados desde un enfoque cualitativo, el cual recupera experiencias finas de la vivencia de los hombres, aportando reflexiones específicas de sus contextos, en particular los contextos socioeconómicos. Por otro lado, los pocos estudios cuantitativos del malestar-bienestar aportan una idea más generalizada sobre el estado de la cuestión en razón a rasgos identitarios con los que los hombres se identifican y los sentimientos que estos les generan. Finalmente, de la inercia de los resultados obtenidos en los textos, queda todavía pendiente los caminos para problematizarlo más allá de solo nombrarlo y adentrarnos en la relación malestar-paternidad, malestar-cuidados y las posibles estrategias de atención a varones que sienten el malestar.

\section{REFERENCIAS}

Abarca, H. (2000). Discontinuidades en el modelo hegemónico de masculinidad. En M. Gogna (Ed.), Feminidades y masculinidades. Estudios sobre salud reproductiva y sexualidad en Argentina, Chile y Colombia (la ed, pp. 193-244). Buenos Aires: Centro de Estudios de Estado y Sociedad.

Aguayo, F., Barker, G. y Kimelman, E. (2016). Editorial: Paternidad y Cuidado en América Latina: Ausencias, Presencias y 
Transformaciones. Masculinities \& Social Change, 5(2), 98-107. doi:10.17583/mcs.2016.2140

Arksey, H. y O'Malley, L. (2005). Scoping studies: towards a methodological framework. International Journal of Social Research Methodology, 8(1), 19-32. doi:10.1080/1364557032000119616

Badinter, E. (1993). XY, La Identidad Masculina. Madrid: Alianza Editorial.

Batthyány, K. (2013). Cuidados, corresponsabilidad y democratización de las relaciones de género. Recuperado de http://www.cde.org.py/ web/attachments/article/153/Conferencia\%20Karina \%20Batthy\%C3\%Alny\%20-\%20VII\%20Encuentro\%20Mujeres \%202013.pdf

Bonino, L. (2000). Varones, género y salud mental: deconstruyendo la "normalidad" masculina. En M. Sagarra y A. Carabí (Eds.), Nuevas Masculinidades (pp. 41-64). Barcelona: Icaria.

Bonino, L. (2002). Masculinidad hegemónica e identidad masculina. Dossiers Feministes. Masculinitats: mites, de/construccions i mascarades, 7-37.

Bonino, L. (2008). Salud, varones y masculinidad. Voces de hombres por la igualdad de género, 182-187.

Burin, M. (2007). Precariedad laboral, masculinidad, paternidad. En M. Burin, M. L. Jiménez e I. Meler (Eds.), Precariedad laboral y crisis de la masculinidad. Impacto sobre las relaciones de género (pp. 87-120). Buenos Aires: Universidad de Ciencias Empresariales y Sociales.

Burin, M. (2010). Género y salud mental: construcción de la subjetividad femenina y masculina. Buenos Aires.

Cantoral, G. (2014). De las desigualdades de género a los malestares. La cotidianidad de mujeres y varones de San Cristóbal de las Casas, Chiapas. Universidad de Ciencias y Artes de Chiapas. 
Connell, R. W. (1997). La orgaización social de la masculinidad. En T. Valdés y J. Olavarría (Eds.), Masculinidad/es, poder y crisis (pp. 31-48). Santiago: Isis y FLACSO Chile.

Connell, R. W. (1998). Masculinities and globalization. Men and

Masculinities, 1(1), 3-23. doi:10.1177/1097184X98001001001

Connell, R. W. y Messerschmidt, J. W. (2005). Hegemonic

Masculinity: Rethinking the Concept. Gender and Society, 19(6),

829-859. doi:10.1177/0891243205278639

De Keijzer, B. (2006). Hasta donde el Cuerpo Aguante: Género,

Cuerpo y Salud Masculina. La manzana, 1(1). Recuperado de http://www.estudiosmasculinidades.buap.mx/paginas/re porteBenodekeiizerhtm

Figueroa, J. G. (2001). La soledad en la paternidad. Fem (publicación feminista mensual), 25(218), 15-48.

Figueroa, J. G. (2007). El derecho a la salud en la experiencia de los varones: ¿un concepto ambivalente en los modelos de masculinidad vigentes? En M. Egúsquiza (Ed.), Exclusión y derecho a la salud La función de los profesionales de la salud (pp. 373-392). Lima: EDHUCASALUD.

Figueroa, J. G. (2013). Algunas reflexiones sobre el estudio de los hombres desde el feminismo y desde los derechos humanos. Estudios Feministas, 21(1), 371-393. doi:10.1590/S0104$\underline{026 \times 2013000100019}$

Figueroa, J. G. (2014). Algunas propuestas dialógicas para relacionar paternidad, salud y mortalidad. Iztapalapa. Revista de Ciencias Sociales y Humanidades, (77), 55-75. doi:10.28928/ri/772014/atc2/figueroapereaig

Fleiz, C., Ito, M. E., Medina-Mora, M. E. y Ramos, L. (2008). Los malestares masculinos: Narraciones de un grupo de varones adultos de la Ciudad de México. Salud mental, 31(5), 381-390.

Gálvez, L. (2013). Una lectura feminista del austericidio. Revista de Economía Crítica, (15), 80-110. doi:10.7868/s0320930x13040130 
González, M. J. y Jurado, T. (2015). Introducción. En M. J. González y T. Jurado (Eds.), Padres y Madres Corresponsables. Una Utopía Real (la ed., pp. 11-29). Madrid: La Catarata.

Herrera, F., Aguayo, F. y Goldsmith, J. (2018). Proveer, cuidar y criar: evidencias, discursos y experiencias sobre paternidad en América Latina. Polis. Revista Latinoamericana, (50), 5-20. Jiménez, M. L. (2008). Reflexiones acerca de la Paternidad. La manzana, 3(4).

Jiménez, M. L. (2013). Reflexiones sobre ser proveedor en la crisis económica y del empleo. Impactos desde la perspectiva de género. En J. C. Ramírez y J. Cervantes (Eds.), Los hombres en México: Veredas recorridas y por andar. Una mirada a los estudios de género de los hombres, las masculinidades (pp. 53-70). Guadalajara: Universidad de Guadalajara.

Jiménez, M. L. (2016). Algunos efectos de los cambios en la economía (trabajo y precarización) en la vida de varones y en sus relaciones de género. En T. E. Rocha y I. Lozano (Eds.), Debates y reflexiones en torno a las masculinidades:

Analizando los caminos hacia la igualdad de género (la ed., pp. 119136). Ciudad de México: Universidad Nacional Autónoma de México.

Jiménez, M. L. y Tena, O. (2007). Algunos malestares de varones mexicanos ante el desempleo y el deterioro laboral. En M. Burin, M. L. Jiménez y I. Meler (Eds.), Precariedad laboral y crisis de la masculinidad. Impacto sobre las relaciones de género (pp. 148173). Buenos Aires.

Juárez, C. y Herrera, C. (2016). Deseando no ser violento: las dificultades para dejar de ser hombre. En T. E. Rocha \& I. Lozano (Eds.), Debate sobre masculinidades. Poder, desarrollo, politicas públicas y ciudadanía. (pp. 102-117). Ciudad de México: Universidad Nacional Autónoma de México. Madrid, S. (2017). The good night kiss: fatherhood among corporate managers and the reconfiguration of hegemonic masculinity in Chile. Norma. International Journal for 
Masculinity Studies, 12(3-4), 240-255.

doi:10.1080/18902138.2017.1362536

Mena, P. (2015). Cuando los varones se quedan con sus hijos:

familias de padres solteros en Querétaro. Revista

Interdisciplinaria de Estudios de Género, 1(2), 111-144.

Mena, P. y Rojas, O. (2010). Padres solteros de la ciudad de

México. Un estudio de género. Papeles de Poblacion, 16(66), 4174 .

Montesinos, R. (2004). La nueva paternidad: expresión de la transformación masculina. Polis, 2(4), 197-220.

Morales, A., Catalán, A. y Pérez, F. (2018). Los padres también se deprimen en el postparto: comprendiendo el fenómeno desde la voz de sus protagonistas. Polis, 17(50), 161-181. doi:10.4067/s0718-65682018000200161

Nieri, L. P. (2017). Relación entre la sensibilidad paterna y los estilos de apego, la personalidad y la capacidad empática, según variables sociodemográficas. Psicología desde el Caribe, 34(1), 1-27.

Núñez, G. (2004). Los "hombres" y el conocimiento. Reflexiones epistemológicas para el estudio de "los hombres" como sujetos genéricos. Desacatos, (15-16), 13-32.

Olarte, C. A. (2016). Emoción y hegemonía, la vivencia masculina en Comunicación. Cinzontle, 8(18), 26-34.

Olarte, C. A. (2017). Masculinidad y afectividad en el espacio público: la percepción de los varones ante las prescripciones sociales de género. Análisis en una universidad de Tabasco. Universidad Veracruzana.

Olavarría, J. (2001). Hombres a la deriva?: poder, trabajo y sexo. FLACSOChile.

Pallahuala, S., Avendaño, S. y Estrada, C. (2011). De la Tradición y la Evolución: Bienestar psicológico en hombres patagónicos contemporáneos. Magallania (Chile), 39(1), 71-81. doi:10.4067/s0718-22442011000100004 
Perujo, E. (2015). Ser padre desde la incertidumbre. Experiencias de paternidad y divorcio de varones de clase media y alta en la Ciudad de México. Trace. Travaux et Recherches dans les Amériques du Centre, 68, 100-124.

Piña, C. (2003). Mujeres que escriben sobre mujeres (que escriben) II. (la ed.). Buenos Aires: Editorial Biblos.

Ramírez, J. C. y Uribe, G. (2008). Masculinidades: el juego de género de los hombres en el que participan las mujeres (la ed.). Guadalajara: Plaza y Valdés Editores.

Ramos, M. (2001). La paternidad y el mundo de los afectos. Fem (publicación feminista mensual), 25(219), 10-14.

Rocha, T. E. (2016). Hombres en transición de roles y la igualdad de género: retos, desafíos, malestares y posibilidades. En T. E. Rocha y I. Lozano (Eds.), Debates y reflexiones en torno a las masculinidades: Analizando los caminos hacia la igualdad de género (pp. 32-45). Ciudad de México: Universidad Nacional Autónoma de México.

Rojas, O. L. (2017). Resistencias masculinas para el avance en la igualdad de género en México. Coyuntura demográfica, 12, 7985.

Salguero, A., Córdoba, D. y Sapién, S. (2014). Dificultades, malestares y quejas de algunos hombres sobre su paternidad. Revista Electrónica de Psicología Iztacala, 17(2), 455480.

Salinas, P. (2007). Los discursos masculinos como en la configuración del liderazgo y dispositivos de control y tensión empoderamiento femenino. Estudios Feministas, 15(3), $336-562$.

Salinas, P. y Arancibia, S. (2006). Discursos Masculinos sobre el Poder de las Mujeres en Chile: Sujetos y Subjetividades. Ultima década, (25), 65-90. doi:10.4067/s071822362006000200004 
Sapién, J., Salguero, M. y Córdoba, D. (2011). Dificultades sexuales vividas por los varones durante el embarazo. Revista Electrónica de Psicología Iztacala, 14(3), 129-155.

Segato, R. (2018). "El problema de la violencia sexual es politico, no moral" | Entrevista a la antropóloga Rita Segato, una estudiosa de la violencia machista. PAGINAI2. Recuperado de https://www.paginal2.com.ar/162518-el-problema-de-laviolencia-sexual-es-politico-no-moral

Solís, L. (1998). Feminismo y malestar masculino. En N. Ramírez (Ed.), VI Symposiium Internacional de Crítica Literaria y Escritura de Mujeres en América Latina (pp. 230-237). Salta: Editorial Universidad Católica de Salta.

Téllez, A. y Verdú, A. D. (2011). El significado de la masculinidad para el análisis social. Revista Nuevas Tendencias en Antropología, (2), 80-103.

Tena, O. (2007). Problemas afectivos relacionados con la pérdida, disminución y riesgo de pérdida del empleo en varones. En M. L. Jiménez y O. Tena (Eds.), Reflexiones sobre masculinidades y empleo (pp. 357-376). Cuernavaca: Universidad Nacional Autónoma de México.

Tena, O. (2010). Estudiar la masculinidad ¿para qué? En N. Blázquez, M. Ríos y F. Flores (Eds.), La investigación Feminista: Epistemologia, metodología y representaciones sociales (pp. 271-292). Ciudad de México: Universidad Nacional Autónoma de México.

Tena, O. (2014). Malestares laborales y condición masculina. Reflexiones en torno a la "flexibilidad laboral". En J. G. Figueroa (Ed.), Políticas públicas y la experiencia de ser hombre: paternidad, espacios laborales, salud y educación (pp. 51-78). Ciudad de México: El Colegio de México.

Tena, O. y Jiménez, L. (2007). Reflexiones sobre masculinidad y empleo. Cuernavaca: Universidad Nacional Autónoma de México, Centro Regional de Investigaciones Multidisciplinarias. 
Tena, O. y Jiménez, M. L. (2014). Algunos malestares en la experiencia de los varones: ¿podemos ir reflexionando sobre sus derechos sexuales y reproductivos? En J. G. Figueroa y A. Salguero (Eds.), Y si hablas de...sde tu ser hombre?: Violencia, paternidad, homoerotismo y envejecimiento en la experiencia de algunos varones (pp. 331-358). Ciudad de México: El Colegio de México.

Torre, E. y Rodríguez, M. (2019). Paternidades a distancia:

Malestares de padres separados de sus hijas e hijos tras la deportación. Estudios Fronterizos, 20(e20), 1-22.

doi:10.21670/ref.1902023

Tovar, D. (2016). Re-significaciones del trabajo y de la provisión económica: masculinidades en hombres de la Ciudad de México. En T. E. Rocha y I. Lozano (Eds.), Debates y reflexiones en torno a las masculinidades: Analizando los caminos hacia la igualdad de género (pp. 138-149). Ciudad de México: Universidad Nacional Autónoma de México.

Tuñón, E. y Bobadilla, D. J. (2005). Mortalidad en varones jóvenes de México. Estudios Sociales, 13(26), 67-84.

Villanueva, C. A. (2015). Las paternidades contemporáneas como espacios de transformación relacional y permisión emocional. Revista de la Facultad de Trabajo Social, 31(31), 109-129. doi:10.18566/rfts.v3ln3l.a06

\section{ACERCA DE LAS AUTORAS}

Carolina Olvera Castillo (carolina.olveracast@gmail.com) es integrante del Observatorio Universitario de Equidad y Género de la Universidad Autónoma de San Luis Potosí. Es Licenciada en Psicología por parte de esta misma Institución y Maestra en Políticas Públicas y Género egresada de la Facultad Latinoamericana de Ciencias Sociales. Candidata a doctora en psicología 
por la Universidad de Guanajuato en el Doctorado Interinstitucional en Psicología (ORCID 0000-0001-5877-1041).

María Gabriela Luna Lara (gabyluna@ugto.mx) es licenciada en Psicología y maestra en Psicología Social por la UNAM. Doctora en Psicología por la Universidad de Barcelona (España). Docente e investigadora en la Universidad de Guanajuato, es especialista en conductas proambientales relacionadas con la problemática de los residuos (consumo, manejo y desecho), en estudios de género y el empleo de métodos de investigación cualitativos (ORCID 0000-0002-4198-3950).

Recibido: 01/10/2019

Aceptado: 01/12/2019

CÓMO CITAR ESTE ARTÍCULO

Olvera Castillo, C. y Luna Lara, G. (2020). El malestar en los hombres. Caleidoscopio - Revista Semestral de Ciencias Sociales y Humanidades, 23(42)., 269-295. doi:10.33064/42crscsh2148 